\title{
Tiamulin fumarat - new makrolide on bovine mastitis therapy in vivo and in vitro
}

\author{
Vera Kriukova*, Nadezda Kuznechova, Viktoriya Gaponova, and Lilia Sabyrzyanova \\ St. Petersburg State academy of Veterinary Medicine, St. Petersburg 196084, Russia
}

\begin{abstract}
Antibiotics nowadays are widely used for the treatment of mastitis in cows, which, along with a positive effect, cause negative consequences to the organism of an animal, can be consumed by people and interacts the cultural qualities of microorganisms However, it is almost impossible to refuse from antibacterial therapy of mastitis with chemotherapy drugs. And the variety range of chemotherapy drugs for mastitis therapy today is very wide. Since we conduct monitoring devoted to full characteristics of the strains of Streptococcus spp., isolated from bovine mastitis milk of the geographical zone of Leningrad region, Russia. That is, we analyses received data on the cultural, microbiological, serological, virulence factors, and sensibility to main used in practice antibiotics and to new ones. Taking into account the already proven resistance of microorganisms to antibiotics, the biological industry offers antibacterial drugs based on new active substances for use in the treatment of cow mastitis. In the assay described in this paper, we set a goal-to investigate the antibacterial effect of tiamulin fumarat to clinical isolates of streptococci received from milk of cows with mastitis, and to determine its clinical efficacy on cows with mastitis of the bacteriological etiology
\end{abstract}

\section{Introduction}

Pathogenic microorganisms play role of the one of the leading factors in the etiology of cattle mastitis. Skin of the udder at normal condition contains Staphylococci spp., as opportunistic microflora, the last easily becomes pathogenic as only natural immunity of an animal decrease and due to their wide distribution in the cowshed environment and the ability to live and reproduce on the skin surface of the teats and udders, provoke inflammation process [1].

According to different microbiological statistical data - microorganisms of Staphylococci spp., Streptococci spp., are isolated in $90 \%$ of cases, in combination with a bacteria of the E. coli group [1]. In our general work, that proceeded this assay, we collected clinical isolates of Streptococcus spp., from bovine mastitis samples of milk. Geographical zone of our scientific interest work is NorthWest area of Russia, Leningrad region.

We concentrated our attention to sterptococci as example of pathogenic microorganism of epidemiological and eizootological importance. It is the causative agent of mastitis in women, as well as cows, sheep, goats and other animals [2]. In humans, it can cause mastitis, pneumonia, urinary tract infections, as well as sepsis and meningitis of newborns. In recent decades Streptococcus spp., attract the attention of physicians as pathogens of pregnant and neonatal infections of newborns. More research is needed on horizontal transmission of Streptococcus spp., between animals and man [3].
Streptococcus spp. - one of the first microorganisms isolated from the milk of cows with udder inflammation, France, 1884. In 1891 Kitt gave the name of the pathogen-S. agalactiae. Later it was found that streptococci are divided into different serological groups. The classification criteria for streptococci species are the structure of the group- polysaccharide, which is part of the cell wall of microorganisms. Immunological group classifications were developed by R. Lancfield in 1933, using capital letters of Latin alphabet from $\mathrm{A}$ to $\mathrm{H}$ and from $\mathrm{K}$ to $\mathrm{T}$. All in all it was 20 serotypes. The main pathogenic Streptococci spp., agents of human and animals belong to groups from A to E [4]. It is known that mastitis process provoke the following groups; streptococci of group B- S. agalactiae and streptococci gr. C-S. dysgalactiae, and streptococci gr. E-S. uberis, streptococci gr. D-S. faecalis [1-3].

Beif characteristic of main pathogenic streptococci: groups: gr. "A"- S. pyogenes. Basically is pathogenic to humans. From animals can be isolated relatively rarely, however, it can cause mastitis in various species of animals, septicemia in birds, pharyngitis, bronchitis and pneumonia in monkeys, Guinea pigs, endometritis in dogs. When pyogenic Streptococcus is isolated from domestic animals, there is often a possibility that the source of the infection is sick or ill person, who was in contact with these animals [5].

Group "B". S. agalactiae. It is the causative agent of mastitis in cows, sheep, goats and other animals. In humans, it can cause mastitis, pneumonia, urinary tract infections, as well as sepsis and meningitis of newborns.

* Corresponding author: Vera.kryukova@inbox.ru 
In recent decades, these microbes have attracted the attention of physicians as pathogens of pregnant women and neonatal infections of newborns. It possesses the typical properties of an obligate parasitic pathogen: does not develop at the environment and preserves in it for a short time periode, has a strong adhesion to the epithelium of mammary ducts, is capable to produce necrotic, hemolytic toxin and hyaluronidase. It can be found rarely in the mammary gland of healthy cows, almost often is released in subclinical mastitis Inflammation of the udder of cows, caused by S. agalactiae, most often begins with the mucous membrane of the milk tank and then spreads through the milk ducts and lymphatic pathways to other parts of the corresponding quarter of the udder. Further spread of the disease usually progresses slowly, and therefore, longterm infection and the inflammatory process in acute phase of clinical and subclinical mastitis can persist. Complete overgrowth of the udder with connective tissue is rare, although the inflammatory process lasts for years [6]. Gr. C - in the etiology of mastitis, only S. dysgalactiae matters. It causes chronic, subclinical mastitis. Such mastitis is less common than mastitis caused by $S$. agalactiae [7].

Streptococci group E-S. uberis - cause subclinical and mild chronic mastitis in cows. In recent years, the role of $\mathrm{S}$. uberis as a causative agent of mastitis has increased dramatically, a number of researchers associate this process with a variety of cultural and biochemical properties of the pathogen and, as a consequence, with its unsatisfactory identification in the past $[1,7,8]$.

Gr. D - Enterococcus. Currently, enterococci belong to a separate genus Enterococcus, according to classification of Bergi. In microbiological study of milk from cows with mastitis, enterococci are isolated very often, since they are usually representatives of the microflora of the digestive tract and exhibit pathogenic properties in an organism with immunity resistance decrease, under unsanitary conditions of animal keeping. In such cases they can cause purulent inflammatory diseases. The genus Enterococcus currently includes 16 species of microorganisms [9, 10]. The most important in the etiology of mastitis are the following: E. faecalis, have a relatively greater pathogenicity compared to other enterococci, has etiological significance in mastitis, otitis, pneumonia, streptococcosis of birds and meningitis in animals; E. faecium is obligate inhabitants of the intestines of animals and humans

Serological distribution of Streptococcus spp., ,causing mastitis in cows, varies from regione to regione, from farm to farm. According to our bacteriological survey-monitoring of the 5 milking farms from the Leningrad region, totally 105 samples of mastitis milk, at the period from 2015-2017 years, predominated Streptococci gr. B (S. agalactiae) and Streptococci gr. E (S uberis). For example, in Baikal region Streptococcus gr. E dominates [8].

Gram "+" pathogenic microorganisms undergoes different mutations in their living process, displace other genera that difference from their biological level, scientist all over the world pronounced such a problem, especially with existing resistance to antibiotics $[1,10]$. Already stated clinical examples of such clinical strains of microorganisms, resistant to antibacterial drugs are increasingly found in the literature. For example, World Health Organization has confirmed the threat of the spread of penicillin-resistant strains of St. Aureus. The selection of bacterial strains with high resistance and pathogenicity can be regarded as the result of the deterioration of the environmental situation and immune reactivity of the body, irrational and unbalanced feeding, uncontrolled widespread use of antibiotics and hormonal drugs for treatment.

Discussion of the problems of the treatment of mastitis in animal. In every manual for animal treatment it is written that mastitis - is a complex problem, that have to be sold step by step. Animal with udder inflammation needs to receive etiotropic and pathogenetic therapies, regarding the origin of pathological agent. All therapies should be combined with constant antiseptic treatment of udder tissue and delete of milking excretions. In order, the chosen treatment of mastitis were successive and quick, the farmer must well know the epizootic situation in his farm and perform constant diagnostic laboratory tests. Especially, emphasize on routine antimicrobial activity tests of pathological agents, isolated in his farm. Unfortunately, no one do such laboratory tests constantly, as do not see any necessity. And unfortunately, currently don't exist any monitoring programs on mastitis on government level and normally, mastitis treatment is started in blind format. Antibacterial drugs are chosen by the main rule - "the wider antibacterial effect is - the better'. Mastitis remedies most often presents forms for intamuscle injections or syringes for intra-mammary duct introduction. Usually, all veterinarians choose for pathogenic therapy of mastitis combined antibiotics, consisting of the following classes of active substances: synthetic penicillins, macrolide and aminoglycosides [11]. Such a chemotherapeutic treatment presents mostly widespread bovine mastitis therapy at the milking farms and it works against main Gram "+" and Gram "-_" microorganisms.

The most active antibacterial agents against staphylococci are enrofloxacin and gentamicin. Among macrolides and lincosamides, thiamulin showed the greatest activity, which was almost as effective as enrofloxacin and gentamicin [12, 13]. Staphylococci, resistant to tetracycline, oxytetracycline and chlortetracycline, as a rule, remain sensitive to doxycycline, which is probably due to the more pronounced lipophilic properties of its molecules, so that the antibiotic is easier to penetrate into the bacterial cell. At the veterinary literature are described different clinical cases of the unsuccessful treatment of bovine mastitis with syntetic penicillin, macrolide or tetracycline $[1,6]$. S However in medical literature resistance genes to the action of erythromycin in streptococci group A and B: mreA and mefA are described [5]. The above genes encode enzymes responsible for modifying the structure of antibiotics through phosphorylation, adenylation, and acetylation of hydroxyl and amino groups. 
Biological industry suggests new antibacterial drugs for pathogenic therapy of bacterial infections of animals, in order to increase the clinical efficacy of existing treatment. For example, such a new active substance is tiamulin fumorat: group - makrolide, semi-synthetic derivate of diterpen antibiotics. On the veterinary market exist several commercial drugs of it [12].

Trial described in this paper was conducted in the framework of scientific survey-monitoring devoted to the study of the microbiological, serological, antibacterial and genetic properties of clinical isolates of Streptococci spp, recieved from mastitis samples of milk, of cows from the milking farms of the Leningrad region, Russia, with the final aim - to develop actual practical recommendations for veterinarians for treatment of bovine mastitis of streptococci etiology.

The aim of the work described in this article is to determine the antimicrobial activity of the new chemotherapeutic substance tiamulin fumarat against clinical isolates of Streptococci spp., collected from milk of cows with mastitis, and clinical effectiveness of it in the treatment of cows, with mastitis of cocci etiology.

\section{Materials and methods}

For our assay were used 25 clinical isolates of Streptococcus spp., this collection is the result of bacteriological analysis of milk samples of cows with mastitis, conducted from 2015-2017 years. In proceeded assay, clinical isolates were serologically typed as Streptococci group B, C and D, using commercial antigen- serums of "AQUAPAST" production. We preserved our clinical isolates in meat-peptone broth media enriched with glucose and dissolved in $1: 1$ proportion with glycerin, at the temperature $-25 \mathrm{C}$ in plastic vials. Such conservation allowed using clinical isolates for different types of samplings: susceptibility to well known and new chemotherapeutic substances, PCR of main known virulence-factors genes.

The active substance of tiamulin fumarat, that we took for assessment, as an example of a new chemotherapeutic agent, presents a semi-synthetic derivate of the diterpene antibiotic pleuromutilin, produced by Pleurotus mutulis. It posesses bacteriostatic activity against both gram-positive and gram-negative microorganisms (Mycoplasma spp., Haemophilus spp., Hyodesenteriae Treponema, Staphylococcus spp., Streptococcus spp., Clostridium perfringens, Leptospira spp., Listeria monocytogenes, Pasteurella spp., Corynebacterium pyogenes [13].

In veterinary medicine, remedies of the group of makrolides are characterised as new chemotherapy drugs, and are used by veterinarians as drugs with $100 \%$ efficacy, cause of the absence of the stated cases of microbial resistance to it. So we decided to test it in vitro, using 25 clinical isolates of streptococci, and in vivo in cows, diagnosed with mastitis. As a working model of tiamulin fumarat we took the commercial drug "Timukotin" ("AVZ" production), available on the market. The last includes two active substances: tiamulin fumarat, in a concentration of $100 \mathrm{mg} / \mathrm{ml}$ and colistin sulfate in a concentration of $180,000 \mathrm{IU} / \mathrm{ml}$, which belongs to the group of polypeptide antibiotics polymyxin E, active only against gram-negative bacteria: Pseudomonas spp., groups of Enterobacteriaceae [14].

Antimicrobial activity of thiamulin fumarate was determined by the method of "Successive serial dilutions", allowing detecting the value of minimum inhibitory concentration on the liquid media, which corresponds to bacteriostatic activity of the drug, according to methodical instructions of MUK 4.2.1899004 [15].

Further, was set a clinical trial of tiamulin fumorat in vivo. We chose two farms of the Leningrad region: farm No. 1 and farm No. 2, in order to study clinical effect of tiamulin fumorat, administered to cows with clinical and subclinical mastitis of cocci etiology in a dosage, following the instruction for "Timukotin". Trial lasted for 1 year 2016-2017.

In each farm were formed experimental and control groups of animals. For control group were chosen 5 animals with mastitis, treated according to generally used scheme at the farm. A total of 25 animals participated at the experiment. $(n=25)$. We tried to select animals with newly identified mastitis without chronic forms. Animals were clinically examined for signs of mastitis and the main requirement were that cases of mastitis were of bacteriology etiology, were isolated Gram positive cocci microflora with gemolysis property, of course, without long lasting detailed typization, as it was impossible to leave animals for a long period untreated.

For in vivo investigation were chosen animals, diagnosed with mastitis, based on clinical survey of animal, and parameters, general condition of the animal, thermometry of udder, inflammation tissue udder reaction, sedimentation milk sampling with $5 \%$ dimastin reagent, automatic calculation of somatic cells, bacteriological examination of milk for the presence of pathogenic cocci microorganisms. During assessment, animals were under daily clinical observation, consisting of the record of the body temperature, respiratory rate and heart rate.

\section{Results and discussion}

As a criterion for assessing the antimicrobial activity of tiamulin fumarat, was calculated the value of its minimum inhibition concentration (MIC) against 25 streptococci isolates, received from the udder secretion of cows with mastitis. As control museum strain of Staphylococcus aureus - 209 P (ATCC) was used. In our study, all isolates were designated by numbers (S. agalactiae No. 25, 30, 31 S. agalactiae No. 2, 4, 5, 20; S. faecalis No. 16, 11, 28). The trial consisted of the following main steps: preparation of 10 vials with meat peptone broth of standard dilutions for each.

Investigated isolates of streptococci, standardization of each vial were done according to the optical density.

Standard on Mcfarland, and was 0.5 standard, which is equivalent to a concentration of $1.5 \times 10(8) \mathrm{CFU} / \mathrm{ml}$. 
Further, we did a parallel 10-fold series of dilutions of the drug, reducing the concentration in each dilution twice. The first dilution (vial) corresponded to the concentration given to us in the drug $100 \mathrm{mg} / \mathrm{ml}$, and was regarded as $100 \%$, and the last, respectively, $10 \%-$ $0.1 \mathrm{mg} / \mathrm{ml}$. Further we inserted $1 \mathrm{ml}$ of each vial with a dilution of a substance to a vial under equal number with Streptococci isolates. All in all we had 25 serials of vials for each testing microorganisms and control serial for St. aureus - 209 P. Each line of serial dilutions were cultivated for 24 hours on thermostat under $37^{\circ} \mathrm{C}$

Table 1. Parameters of clinical survey of animals from experimental and control groups with mastitis

\begin{tabular}{|c|c|c|c|}
\hline \multirow[b]{2}{*}{ № } & \multirow[b]{2}{*}{ Parameters } & \multicolumn{2}{|c|}{ Groups } \\
\hline & & $\begin{array}{l}\text { Experimental } \\
(\mathrm{n}=25)\end{array}$ & $\begin{array}{l}\text { Control } \\
(\mathrm{n}=5)\end{array}$ \\
\hline \multirow[t]{2}{*}{1} & $\begin{array}{l}\text { Clinical signs of udder } \\
\text { inflammation (local } \\
\text { temperature, status of } \\
\text { supramental lymph nodes): }\end{array}$ & 23 & 5 \\
\hline & Subclinical mastitis & 2 & 0 \\
\hline 2 & $\begin{array}{l}\text { Positive sampling of milk } \\
\text { with } 5 \% \text { dimastine }\end{array}$ & 25 & 5 \\
\hline 3 & $\begin{array}{l}\text { Positive reaction of } \\
\text { sedimentation }\end{array}$ & 25 & 5 \\
\hline 4 & $\begin{array}{l}\text { Somatic Sell Count } \\
\text { (thousands } / \mathrm{ml} \text { ) }\end{array}$ & $\begin{array}{c}2500 \\
700 \pm 78.8^{*}\end{array}$ & $\begin{array}{c}2000 \\
000 \pm 10.2\end{array}$ \\
\hline 5 & $\begin{array}{l}\text { Presence of Gram + cocci } \\
\text { m/o with gemolysis } \\
\text { properties }\end{array}$ & Presence & Absence \\
\hline
\end{tabular}

$\mathrm{P} \leq 0.05$ - in comparison to control group

The average resulting value of the minimum inhibitory concentration of tiamulin fumarat at the broth with cultivated microorganisms corresponded to the dilution of $12.5 \%$, which is equal to $12.5 \mathrm{mg} / \mathrm{ml}$ of tiamulin fumarat. For control microorganism - the resulting value was the same.

Recieved value is equivalent to the minimum inhibitory concentration of tiamulin fumarat in composition of the drug "Timukotin", required to suppress the growth of pathogenic cocci microorganisms.

Clinical efficacy of the active substance tiamulin fumarat, as the composite of the drug "Timukotin", was tested on the cows, diagnosed with mastitis, not in a chronic form.

Starting parameters of clinical survey of animals for designation of experimental and control groups are described in table 1.

In all animals in inflammation process was involved 1 or 2 udder lobes.

The drug was injected to animals from experimental groups intramuscularly at a dose of $50 \mathrm{ml}$ once a day for 4 days, according to the drug instructions of $1 \mathrm{ml}$ per $10 \mathrm{~kg}$. Injections were made in different places. Control groups of animals were treated according to the scheme adopted in the farms, with a chemo-therapeutic drug of penicillin group.

During the assay were measured general clinical condition parameters of the animals of the experimental and control groups after 3, 12, 24, 48, 72, 96, 110 hours for validation of any possible toxicological effect of tiamulin fumarat on animals with mastitis It was found that body temperature, pulse rate, number of respiratory movements, rumen frequency rates during treatment in animals of experimental and control groups remained within the physiological norms.

After the end of the 4-th days injection course of the drug, clinical examination of the animals from experimental and control groups was carried out.

$90 \%$ of cows from the experimental group with clinical forms of mastitis showed positive tendencies of treatment: local temperature of the symmetrical quarters of the udder normalized; consistency, mobility and magnitude of supramental lymph nodes corresponded to the norm. Reaction with $5 \%$ dimastin was negative in $90 \%$ of cows. The number of somatic cells in these animals decreased, and was at the average level of $450000 \pm 250.8 \mathrm{ml}$ in milk sample. Bacteriological testing showed visual absence of gram + gemolytical cocci in $80 \%$ of samples.

$100 \%$ of the animals from the control group showed the improvement of inflammatory reaction of the udder. The level of somatic cells decreased till the level of $550000 \pm 48 \mathrm{ml}$. Bacteriological testing showed visual absence of gram positive gemolytical cocci in $100 \%$ of samples.

\section{Conclusion}

Antimicrobial activity of tiamulin fumarat against clinical isolates of pathogenic Streptococcus spp., was $12.5 \mathrm{mg} / \mathrm{ml}$. It is the in vitro confirmation of susceptibility of streptococci to new substance form the group makrolides.

In vivo assessment of tiamulin fumarat, as composite of the drug "Timukotin" demonstrated $90 \%$ efficacy in clinical cases of bovine mastitis, with cocci etiology.

\section{References}

1. A.V. Gorbenko, D.V. Gadzevich, S.A. Guzhvinskaya, O.V. Gadzevich, T.V. Krivogina, Yu.K. Dunaev, Pathogens of clinical and subclinical mastitis of cows and their sensitivity to antibacterial drugs, Vet. Med., 97, 176 (2013)

2. M.N. Isakova, M.V. Ryapsova, N.A. Bezborodova, O.A Bricina, Microbiological background for inflammation of the breast in highly productive cows, Probl. on Vet. Sanit., Hyg. and Ecol., 22, 63 (2017)

3. H.J. Andersen, L.H. Pedersen, F.M. Aarestrup, M. Chriél, Mastitis Pathogens, Contagious Pathogens, J. of Dairy Sci., 86, 1233 (2003)

4. K.V. Shpinev, O.I. Krechikova, V.A. Krechikov, R.S. Kozlov, Streptococcus pyogenes: microorganism characteristics, isolation, identification and determination of sensitivity to antibacterial drugs, Clinic. Microb. and Antimic. Chem., 9, 104 (2007)

5. E.D. Dawson, A.W. Taylor, J.A. Smagala, K.L. Rowlen, Molecular detection of streptococcus 
pyogenes and streptococcus dysgalactiae subsp, Equisimilis. Molec. Biotechn., 42, 117 (2009)

6. M.N. Chelnokova, N.A. Shcherbakova, Diagnosis and therapy of mastitis in cows, News of the Velikiye Luki State Agricult. Acad., 1, 20 (2018)

7. L.I. Smirnova, A.A. Sukhinin, E.I. Prikhodko, I.M. Dorodnaja, Differentiation streptococci isolated from the milk of cows in mastitis, Regulatory issues in vet. med., 4, 136 (2014)

8. A.M. Ablov, E.V. Anganova, A.S. Batomunkuev, The Streptococcosis of Animals and Birds and Specific Characteristics of Pathogens in the Baikal Region, Notes of the Irkutsk st. univ. Ser. Biol. ecol., 11, 105 (2015)

9. E.V. Naumkina, O.A. Abrosimova, E.V. Pakhalkova, N.A. Rogatykh, A.Yu. Mironov, The infections induced by streptococcus of serogroup B in pregnant women, puerpera and newborns, Clin. Lab. Diagn., 61, 107 (2016)

10. O.A. Chuchukina, I.A. Bochkov, State-of-the-art of serogroup B streptococcus-induced infections at the present stage, Epidemiology and infectious diseases, Hot issues, 5, 51 (2013)
11. L.V. Lartseva, O.V. Obukhova, A.N. Barmin, Ecological and biological risk of opportunistic pathogenic microflora resistance to antibiotics (an overview), Russ. J. of Applied Ecol., 47 (2015)

12. S.V. Rusakov, A.Z. Zhuravleva, M.V. Kuznetsova, Comparative study pharmacokinetics Kolimutin company "SP VETERINARIA SA" Timukotin solution and production LLC "NEC" AGROVETZASCHITA, Regulat. issues in veter. med., 1, 41 (2010)

13. P.S. Lobova, V.E. Abramov, A.V. Morosova, A.Yu. Guliaeva, N.I. Tiagnibedina, B.V. Violin, Sensitivity of staphylococcus filed isolates to antibiotics, Agric. scien., 11, 26 (2009)

14. Instructions for the use of Timukotin for the treatment of cattle and pigs for diseases of bacterial and mycoplasma etiology, approved by the deputy head of the Rosselkhoznadzor of no. 15, 2013, registration no. 77-3-29.13-1654 No.PVR-3-5.8 / 02179 .

15. Determination of the sensitivity of microorganisms to antibacterial drugs, guidelines (Federal Center for State Sanitary and Epidemiological Supervision of the Ministry of Health of Russia, Moscow, 2004) 